\title{
Nursing Employment Trends
}

Regulated nurses - registered nurses (RN), licenced practical nurses (LPN) and registered psychiatric nurses (RPN) - represent the single largest group of professionals in Canadian health care, accounting for almost half of the health workforce. As such, shifts in their employment can have a significant effect on the health care system.

Here are some important facts about the nursing employment situation in 2014.

In 2014, the supply of regulated nurses in Canada declined for the first time in 2 decades.

- The supply of regulated nurses declined by $0.3 \%$ over the previous year.

- The supply of registered nurses faced its first decline (-1.0\%) in almost 20 years.

- There were 406,817 regulated nurses in 2014: 293,205 RNs, 107,923 LPNs and 5,689 RPNs.

- There had been 345,845 in 2005: 268,397 RNs, 72,421 LPNs and 5,027 RPNs.

Growth of the regulated nursing workforce in Canada has remained stable over the last 10 years.

- In 2014, the regulated nursing workforce reached 383,949 .

The number of regulated nurses not renewing their registration exceeded the number of nurses entering the profession.

- Last year saw a net loss of 2,360 nurses eligible to work in the profession.

- The majority of regulated nurses who allowed their registration to lapse were from Ontario.

Nurse retention is highest among regulated nurses employed in hospital setting

- Between 2005 and 2014, more than $80 \%$ of regulated nurses remained in the same workplace setting from one year to the next.

- Close to $90 \%$ of regulated nurses working in a hospital setting were retained.

Note the term "supply" refers to regulated nurses who are eligible to practise in the given year (including those employed and those not employed at the time of registration), while the term "workforce" refers to only those regulated nurses who were employed in their profession at the time of annual registration. 